\title{
Interactions between mastication and stress
}

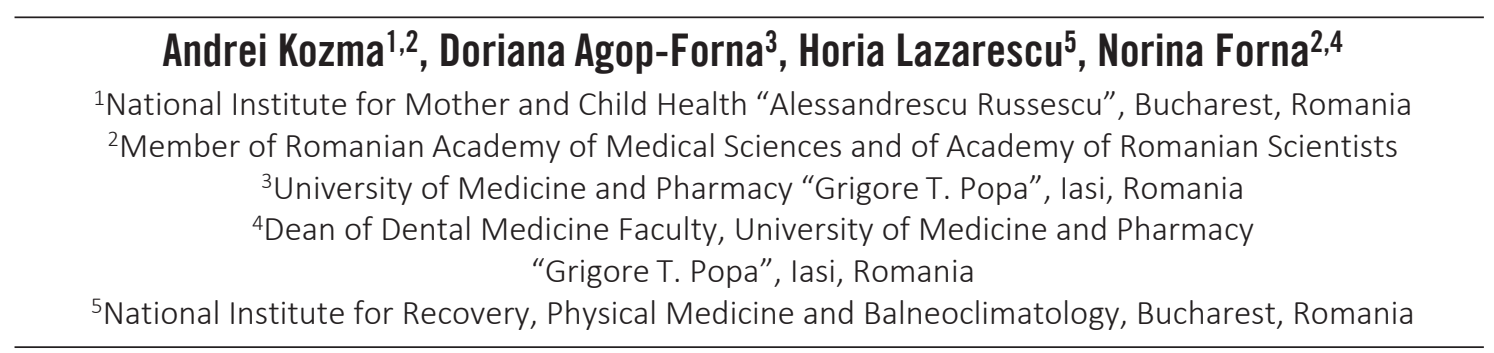

\begin{abstract}
The main neuroendocrine response to stress is via activation of the hypothalamic-pituitary-adrenal (HPA) axis that contribute to the release of glucocorticoid hormone and ACTH. The activation of the HPA axis is a physiological reaction related to stress adaptation but repeated or prolonged HPA axis hyperactivity induces various physiological and psychological disorders. Mastication attenuates stress-induced disorders, cognitive and psychological impairment via suppression of stress-induced activation of the hypothalamic-pituitary-adrenal axis and autonomic nervous system. Chewing induces the increase of the histamine level in the hippocampus that contribute to the recovery of stress-attenuated $\mathrm{N}$-methyl-D-aspartate receptors and the stimulation of the neural centers on long-term. The measuring of stress markers in saliva (alpha-amylasesympathetic nervous system marker, salivary cortisol - endocrine marker, immunoglobulin A-immune system marker) facilitates the assessment of the role of mastication in the stress level attenuation.
\end{abstract}

Keywords: mastication, stress, HPA axis, histamines, salivary markers

Exposure to chronic stress induces various physical and mental effects that may ultimately lead to disease. Among the factors that influenced the susceptibility to stress are genetic vulnerability, coping style, type of personality and social support (1). Considering that stress-related disease (suppression of the immune system, higher risk of viral infection, risk for diabetes mellitus, stress ulcers, ulcerative colitis, mental diseases) has become a global health problem, researchers are focused on various simple and effective strategies to reduce chronic stress in daily life. One of the most sensitive brain areas to stress is the hippocampus, being one of the first brain regions structurally and functionally modified by stress. The increase of corticosterone levels induced by stress can alter hippocampal-dependent cognitive functions as well as psychological behavior. The chronic stress can be produced by occlusal disorders leading to pathologic changes in the hippocampus. Altered mastication due to occlusal disharmony decreases cellular proliferation in the hippocampal dentate gyrus (2).
Mastication represents a protective mechanism against chronic stress as stimulation of the blood supply in neuronal areas is related to the stimulation of the dental baroreceptors, alveolar baroreceptors and masticatory muscles (3). Researches based on Doppler analysis, magnetic resonance imaging and positron tomography demonstrated that during mastication there is an increase of the blood supply to neural areas like sensor-motor areas, insulae, cerebellum, striatum, caudal nucleus, thalamus and temporal lobes $(4,5,6,7,8)$. Regarding the neuronal mechanisms that underlie the interactions between masticatory function and stress-coping behaviors in animals and humans, one review concluded that chewing attenuates stress-induced disorders, such as gastric ulcers, and cognitive and psychological impairment in rodents via suppression of stress-induced activation of the hypothalamic-pituitary-adrenal axis and autonomic nervous system (9). During mastication there is an activation of the histaminergic nervous system to attenuate the stress-induced cognitive deficits. In 
humans, many studies support an association between stress and sleep bruxism. Also gum chewing during stress may affect the levels of various stress markers in the saliva and plasma. One investigation of the reduction of the stress during mastication was performed by measuring the plasma corticosterone levels, spatial learning ability, and cell proliferation in the hippocampal dentate gyrus of the adult offspring by allowing pregnant mice to chew on a wooden stick during stress. The conclusion of this study was that allowing mouse dams to chew on a wooden stick during exposure to prenatal stress reduced the increased levels of the prenatal stress-induced plasma corticosterone levels (10). Kubo et al. (2018) examined the effects of maternal chewing on arginine vasopressin expression in pregnant mice exposed to restraint stress. Mice in the test group (stress/chewing group) were given a wooden stick to chew during restraint stress.

The stress increased significantly the secretion of arginine vasopressin in the paraventricular nucleus of the hypothalamus in the dams, while mastication during stress prevented the increase of the arginine vasopressin levels and prevented the increase in plasma corticosterone in the dam by inhibiting the hypothalamic-pituitary-adrenal axis activity. The conclusion of this study was that mastication during induced stress is an effective stress-coping method (11). Rats allowed to chew on a wooden stick while being exposed to restraint stress exhibits the reduction of the levels of suppressed stress-induced noradrenaline release in the amygdala, while non-functional masticatory activity during the stress attenuates the increase in striatal dopaminergic neurotransmission induced by the stressor (11). One research group aimed to test the hypothesis that maternal active mastication influences the effect of prenatal stress on bone mass and bone microstructure in adult offspring, by comparing two groups of pregnant mice, with one group of mice in stress being allowed to chew a wooden stick during the restraint stress period. Prenatal stress was associated to a significant decrease of trabecular bone mass of the offspring, while maternal active mastication during prenatal stress attenuated the reduced bone formation and increased bone resorption, improved the trabecular bone volume as well as the bone microstructural deterioration induced to offspring by prenatal stress. The conclusion of this study was that maternal active mastication during prenatal stress can ameliorate prenatal stress and the consequences on bone mass and microstructure in adult offspring (12).

Modern researches using Transmission Doppler analysis (TDA) showed the increase of carotid blood flow volume during gum chewing (figure 1) (13).

The main neuroendocrine response to stress is via activation of the hypothalamic-pituitary-adrenal (HPA) axis (14). The central element of HPA axis is the hypothalamic paraventricular nucleus responsible for the secretion of corticotropin-releasing hormone and arginine vasopressin. Corticotropin-releasing hormone and hypothalamic paraventricular nucleus have a role in the promotion of the secretion of adrenocorticotrophic hormone. Adrenocorticotropic hormone has a major role in the stimulation of the secretion of glucocorticoid from the adrenal cortex. Glucocorticoid hormones act initiates metabolic, physiological and behavioral actions and modulate many physiological processes, including intermediary metabolism, immune function, skeletal growth, cardiovascular function, reproduction, and cognition. Glucocorticoid in excess causes hyperglycemia and insulin resistance and can induce inflammatory bowel diseases. The activation of the HPA axis is related to stress adaptation but repeated or prolonged HPA axis hyperactivity induces various physiological and psychological disorders (15). Studies in rats and mice, showed that chewing or biting on wooden sticks under immobilization, or cold exposure attenuates the secretion of adrenocorticotropic hormone and plasma corticosterone levels. The sup-

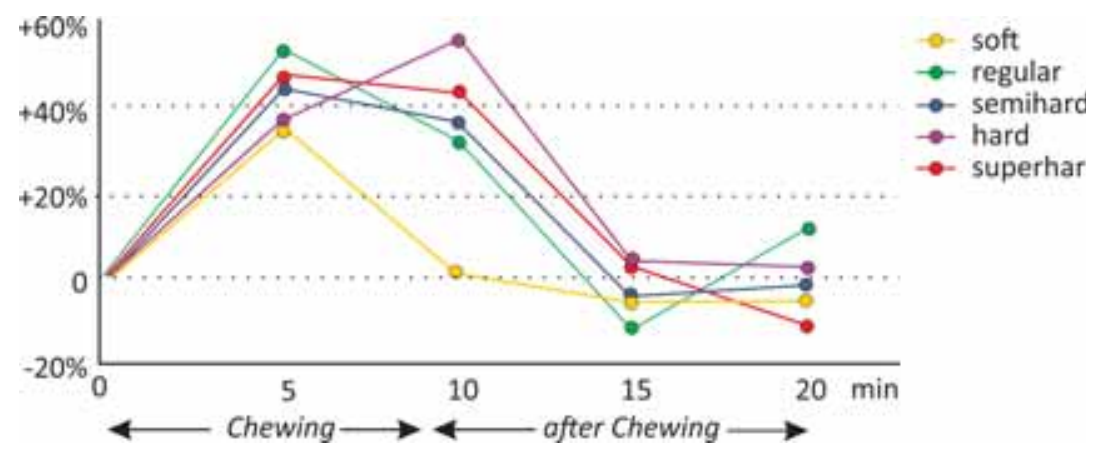

FIGURE 1. Changes of carotid blood flow volume (CCBFV) during gum chewing (related to gum hardness) 
pression of adrenocorticotropic secretion conducts to the decrease of the physiologic stress markers in the paraventricular nucleus of the hypothalamus.

The effects produced by the masticatory stimulation on HPA axis activity via the hippocampus are influenced by various pathways, including the peripheral sensory nervous inputs, stress hormones and their receptors, neurotransmitters and neuropeptides, as well as the autonomic nervous system (16). The disfunction of the masticatory function activates the HPA axis and increases circulating levels of glucocorticoids that disrupts the negative feedback system of the HPA axis, conducting to further enhancing of the secretion of glucocorticoids. The occlusal disharmony induced in mice by the bite-raising procedure, with vertical dimension of the bite raised by approximately $0.1 \mathrm{~mm}$ by applying ultraviolet-ray polymerization resin to the upper molars after treatment conducts after 8 days to the activation of HPA axis and the increase of the circulating corticosterone levels are increased, with significant increases detected only in old bite-raised mice (17). On the other part, the masticatory stimulation during stressful conditions suppresses the hyperactivity of the HPA axis via GCs and GC receptors within the hippocampus, conducting to the amelioration of the chronic stress-induced hippocampus-dependent cognitive deficits (18).

Another neural potential mechanism implied in the release of stress during chewing is the brain histaminergic reaction. It seems that mastication increase histamine $\mathrm{H} 1$ in hippocampus, that contribute to the recovering of $\mathrm{H} 1$ receptor stress-suppressed synaptic plasticity (19). As mastication increases the hypothalamic histamine concentration, it stimulates histaminergic neurons in the TMN, and the electrical stimulation of the TMN facilitates extracellular concentrations of histamine in the hippocampus. Following this mechanism, chewing induces the increase of the histamine level in the hippocampus that contribute to long-term neural potentiation via the recovery of stress-attenuated $\mathrm{N}$-methyl-D-aspartate receptors (19).

One research found that chewing gum contributes to the decrease of stress, anxiety, depression and fatigue both at work and at home as well as to higher well-being. It was suggested that chewing can be compared to meditation practices recommended for stress relief as repetitive activity plays an important role in the stress attenuation (20).

Regarding the effects of the mastication on psychological stress, a research found significant correlations between masticatory ability and depression, anxiety, and stress, as the subjects with high masticatory ability were associated to lower scores for presence of depression, anxiety and stress. This research found an association between low masticatory ability with anxiety, depression, and stress (21).

The measuring of stress markers in saliva (alpha-amylase- sympathetic nervous system marker, salivary cortisol- endocrine stress marker, immunoglobulin A-immune stress marker) facilitates the assessment of the mastication effects in the stress attenuation. To assess stress, three stress salivary markers can be evaluated: alpha-amylase activity, salivary cortisol levels, and secretory immunoglobulin A. Chewing and light teeth-clenching after stress loading lead to a rapid reduction of alpha-amylase activity (22) and salivary cortisol levels (23). The fast chewing rate induces a higher decrease of psychological stress than slow chewing rate (24), and a strong chewing force induces a greater reduction in the mental stress than weak chewing force (25). The time of mastication influences the response of the endocrine system to psychological stress, as continuous chewing for more than $10 \mathrm{~min}$ utes has the highest effectiveness in the reduction of the stress marker levels in saliva (26).

\section{CONCLUSION}

Since 1990s, due to researches performed by doppler transmission analysis, and positron tomography, mastication has been suggested to increase cerebral flow and neuronal activities in various regions of the human brain. The increase of cerebral flow and the suppression of stress-induced activation of the hypothalamic-pituitary-adrenal axis recommend mastication as an additional stress-coping strategy. New researches, based on modern neuroimaging techniques correlated with assessment of saliva stress markers, can clarify the benefits of gum chewing in various stress-related disorders.

\section{Compliance with Ethics Requirements}

The authors declare no conflict of interest regarding this article.

The authors declare that all the procedures and experiments of this study respect the ethical standards in the Helsinki Declaration of 1975, as revised in 2008 (5), as well as the national law. Informed consent was obtained from the patient included in the study.

No funding for this study.

\section{Acknowledgement}

All authors have same contribution for this article. 


\section{REFERENCES}

1. Mohd. Razali Salleh. Life Event, Stress and Illness. Malays J Med Sci. 2008 Oct; 15(4): 9-18.

2. Mori D, Katayama T, Miyake H, Fujiwara S, Kubo KY . Occlusal disharmony leads to learning deficits associated with decreased cellular proliferation in the hippocampal dentate gyrus of SAMP8 mice. Neurosci Lett. 2013 Feb 8; 534:228-32.

3. Firu P, Forna NC. Dinţii în antropologia medical dentară. Edit.PIM, lasi, 2015.

4. Hasegawa Y, Ono T, Hori K, Nokubi T. Influence of human jaw movement on cerebral blood flow. J Dent Res. 2007 Jan; 86(1):64-8.

5. Hasegawa Y, Tachibana Y, Sakagami J, Zhang M, Urade M, Ono T. Flavor-Enhanced Modulation of Cerebral Blood Flow during Gum Chewing. PLoS One. 2013 Jun 19;8(6):e66313. doi: 10.1371/journal. pone.0066313. Print 2013.

6. Hasegawa Y, Ono T, Sakagami J, Hori K, Maeda Y, Hamasaki T, Nokubi T. Influence of voluntary control of masticatory side and rhythm on cerebral hemodynamics. Clin Oral Investig. 2011 Feb;15(1):113-8. doi: 10.1007/s00784-009-0338-5.

7. Zhang M, Hasegawa Y, Sakagami J, Ono T, Hori K, Maeda Y, Chen YJ. Effects of unilateral jaw clenching on cerebral/systemic circulation and related autonomic nerve activity. Physiol Behav. 2012 Jan 18; 105(2):292-7.

8. Nakata M.. Masticatory function and its effects on general health. Int Dent J. 1998;48(6):540-8.

9. Kin-ya Kubo, Huayue Chen, Xiaolin Zhou, Jian-Hua Liu, Olivier Darbin. Chewing, Stress-Related Diseases, and Brain Function. Biomed Res Int. 2015; 2015: 412493. Published online 2015 May 20. doi: $10.1155 / 2015 / 412493$

10. Kin-ya Kubo, Mitsuo linuma, Huayue Chen. Mastication as a Stress-Coping Behavior. Biomed Res Int. 2015; 2015: 876409. Published online 2015 May 18. doi: 10.1155/2015/876409.

11. Kin-ya Kubo, Mika Kotachi, Ayumi Suzuki, Mitsuo linuma, Kagaku Azuma. Chewing during prenatal stress prevents prenatal stressinduced suppression of neurogenesis, anxiety-like behavior and learning deficits in mouse offspring. Int J Med Sci. 2018; 15(9): 849-858

12. Kagaku Azuma, Minori Ogura, Hiroko Kondo, Ayumi Suzuki, Sakurako Hayashi, Mitsuo linuma, Minoru Onozuka, Kin-ya Kubo. Maternal Active Mastication during Prenatal Stress Ameliorates Prenatal Stress-Induced Lower Bone Mass in Adult Mouse Offspring. Int J Med Sci. 2017; 14(4): 348-355.

13. Suzuki M, Ishiyama I, Takiguchi $T$, Ishikawa $H$, Suzuki $Y$, Sato $Y$. Effects of gum hardness on the response of common carotid blood flow volume, oxygen uptake, heart rate and blood pressure to gum-chewing. J Mastica Health Sci; 1994; 4:9-20.
14. Herman JP, McKlveen JM, Ghosal S, Kopp B, Wulsin A, Makinson R, Scheimann J, Myers B. Regulation of the Hypothalamic-PituitaryAdrenocortical Stress Response. Compr Physiol. 2016 Mar 15; 6(2):603-21.

15. Chrousos GP. Stress and disorders of the stress system. Nat Rev Endocrinol.2009;5(7):374-81.

16. Kagaku Azuma, Qian Zhou, Masami Niwa, Kin-ya Kubo. Association between Mastication, the Hippocampus, and the HPA Axis: A Comprehensive Review. Int J Mol Sci. 2017 Aug; 18(8): 1687.

17. Miyake H, Mori D, Katayama T, Fujiwara S, Sato Y, Azuma K, Kubo KY. Novel stress increases hypothalamic-pituitary-adrenal activity in mice with a raised bite. Arch Oral Biol. 2016 Aug; 68:55-60.

18. Ono Y, Kataoka T, Miyake S, Cheng SJ, Tachibana A, Sasaguri KI, Onozuka M. Chewing ameliorates stress-induced suppression of hippocampal long-term potentiation. Neuroscience. $2008 \mathrm{Jul}$ 17;154(4):1352-9.

19. Ono Y, Kataoka T, Miyake S, Sasaguri K, Sato S, Onozuka M. Chewing rescues stress-suppressed hippocampal long-term potentiation via activation of histamine $\mathrm{H} 1$ receptor. Neurosci Res. 2009 Aug; 64(4):385-90.

20. Smith AP, Chaplin K, Wadsworth E. Chewing gum, occupational stress, work performance and wellbeing. An intervention study. Appetite. 2012 Jun; 58(3):1083-6.

21. Hamidreza Roohafza, Hamid Afshar, Ammar Hassanzadeh Keshteli, Mohamad Javad Shirani, Parastu Afghari, Amrita Vali, Peyman Adibi. Masticatory ability with depression, anxiety, and stress: Does there exist any association? Dent Res J (Isfahan) 2016 May-Jun; 13(3): 211-216.

22. Nakajo N., Tomioka S., Eguchi S., Takaishi K., Cho G., Sato K. Gum chewing may attenuate salivary alpha-amylase of psychological stress responses. Journal of Japanese Dental Society of Anesthesiology. 2007;35(3):346-353.

23. Tahara Y, Sakurai K, Ando T. Influence of chewing and clenching on salivary cortisol levels as an indicator of stress. J Prosthodont. 2007 Mar-Apr; 16(2):129-35.

24. Tasaka A, Tahara Y, Sugiyama T, Sakurai K. Influence of chewing rate on salivary stress hormone levels. Nihon Hotetsu Shika Gakkai Zasshi. 2008 Oct; 52(4):482-7.

25. Soeda R, Tasaka A, Sakurai K. Influence of chewing force on salivary stress markers as indicator of mental stress. J Oral Rehabil. 2012 Apr; 39(4):261-9.

26. Tasaka A, Takeuchi K, Sasaki H, Yoshii T, Soeda R, Ueda T, Sakurai $\mathrm{K}$. Influence of chewing time on salivary stress markers. J Prosthodont Res. 2014 Jan; 58(1):48-54. 